\title{
Compliance with iron-folic acid (IFA) therapy among pregnant women in an urban area of south India
}

Mithra P, Unnikrishnan B, Rekha T, Nithin K, Mohan K,

Kulkarni V, Holla R, Agarwal D

Department of Community Medicine, Kasturba Medical College (Manipal University), Mangalore; India

\begin{abstract}
Background: Anaemia is highly prevalent among pregnant women and iron deficiency is the most important cause. Like many other countries, India has policies to give pregnant women iron supplements. Non-compliance is one important challenging factor in combating anaemia.

Objective: To estimate the compliance for IFA tablets among pregnant women and to study the social factors influencing it.

Methodology: This study included 190 pregnant women seeking ante-natal care in tertiary health Centres in the Mangalore city in South India. After Institutional Ethics Committee (IEC) approval, data was collected by personal interview. Missing $\geq 2$ doses consecutively was considered non-compliance. The data was analyzed using SPSS (Statistical Package for Social Sciences) version 11.5.

Results: The mean age of the study population was 25.8 years (SD: 4.1). Majority of the subjects consumed mixed diet and $72.1 \%$ belonged to lower socioeconomic status. Overall, compliance with IFA tablets was $64.7 \%$. Compliance increased with the increase in age, birth order and single daily dose. Forgetfulness and both perceived as well as experienced side effects of IFA therapy were the important factors for non-compliance.

Conclusion: There was a moderate level of Compliance towards IFA tablets with key social and demographic factors playing important role.
\end{abstract}

Keywords - compliance, iron, folic acid, tablets, demographic, social

African Health Sciences 2014;14(1): 255-260 http://dx.doi.org/10.4314/ahs.v14i1.39

\section{Introduction:}

Anaemia is a condition in which the oxygen carrying capacity of the red blood cells is insufficient to meet the body physiological needs. It can cause various health complications in every age group including pregnant women, wherein it can lead to adverse maternal and foetal outcomes ${ }^{[1]}$. It is estimated that as many as $20 \%$ of maternal deaths are directly caused by anaemia through its complications and it is an associated cause in as many as $50 \%$ of maternal deaths worldwide ${ }^{[1],[2]}$. The World Health Organization (WHO) defines anaemia as blood hemoglobin concentration less than $11 \mathrm{gm} / \mathrm{dl}$ or hematocrit less than $37 \%$ in pregnant women ${ }^{[1]}$. The WHO estimates that $58 \%$ of the women in developing countriesare anaemic ${ }^{[2]}$.

\section{Corresponding Author: \\ Prasanna Mithra P. \\ Community Medicine,Kasturba Medical \\ College, Manipal University \\ Light house hill road, Mangalore \\ Karnataka State - 575001 India \\ Mob: +919449243260 \\ Email:ppmithra@gmail.com}

The prevalence of anaemia among pregnant women in Karnataka State in India is $62.6 \%$ [3]. Iron deficiency is thought to be the most common cause of anaemia globally, accounting for more than half of anaemia cases in pregnancy ${ }^{[1]}$.

Iron, is an essential nutrient, which is required for haemoglobin synthesis, other than the normal wellbeing, whose demand increases highly during pregnancy and many times does not get supplied through the regular diet. This can be worsened by the loss of appetite during pregnancy ${ }^{[4]}$. Therefore the most suitable mass intervention for iron supplementation is administering Iron along with Folic acid in the form of tablets to pregnant women aimed at increasing the haemoglobin concentration, so that the level of anaemia at term could be reduced to the best possible extent ${ }^{[5],[6],[7]}$.

Most Ministries of Health in developing countries have policies to give pregnant women either iron by itself or combined with folic acid in tablet form (IFA tablets). The National protocols in India require the provision of 1 tablet containing $100 \mathrm{mg}$ elemental iron and 0.5 $\mathrm{mg}$ folic acid for daily consumption to all women during pregnancy for 100 days. 
But effectiveness and success of such interventions depend on the compliance to the Iron-folic acid tablets. Compliance describes the degree to which a patient correctly follows a medical advice. Many experts believe that one of the main reasons that national iron supplementation programs have failed is women's "noncompliance" [1],[5]. There are factors including health system and patient factors which determine the compliance, which are not studied extensively [5],[6] There are no clear cut offs for non-compliance. Missing 2 or more doses consecutively is usually considered as non-compliance ${ }^{[4]}$.

Thus compliance is essential for Iron Folic Acid therapy which in turn is influenced by several social and demographic factors. This study was carried out in a tertiary care centers in Mangalore to assess the awareness, practices, level of compliance and the factors affecting the level of compliance among the pregnant women with iron folic acid therapy.

\section{Materials and Methods:}

This hospital based cross sectional study was carried out in the Lady Goschen Hospital and Kasturba Medical College Hospital Attavar, which are tertiary care centers providing Mother and Child Health services affiliated as teaching hospitals to Kasturba Medical College (Manipal University) Mangalore. The study was carried out for a period of two months (January-February 2012). The pregnant women attending the Antenatal Care clinic in the study hospital during the study period were included as the subjects in the study. The sample size was calculated using anticipated 33\% non-compliance with IFA tablets ${ }^{[4]}$, with $7 \%$ absolute precision, 95\% confidence interval and 10\% non-response error, as 190.

After obtaining clearance from the Institutional Ethics Committee (IEC) and permission from the Hospital Authorities, the hospitals were visited for data collection. The subjects were selected using non-random sampling method (convenient sampling) till the required sample size of 190 was reached. The study subjects were explained about the objectives of the study in their vernacular language in clear words, were provided with the information sheet and a written informed consent was obtained from each of the subjects. Those who were not willing to participate were excluded from the study. The data was collected by using a pretested, semi- structured proforma, which included the demographic information, questions on practices related to Iron Folic Acid therapy, the number of doses including therapeutic purposes, their compliance and factors related to missing of the doses, followed by observing their latest hemoglobin levels from their records at the time of the interview. The socioeconomic status was calculated using Modified Kuppuswamy Scale ${ }^{[]]}$and for analyses, the classes were resorted as Upper and Lower. Missing of two or more doses of IFA was considered as non-compliance ${ }^{[4]}$. Hemoglobin level was classified according to the WHO recommendations ${ }^{[1]}$.

The collected data was coded and entered into the SPSS (Statistical Package for Social Sciences) version 11.5. The results were expressed as proportions. To compare the differences across the groups, chi square test was used and $\mathrm{p}$ value $<0.05$ was considered statistically significant.

\section{Results:}

The study included 190 women attending the Antenatal Clinics. The socio-demographic characteristics associated with the compliance towards consumption of IFA tablets is depicted in Table1. Among the study participants, majority (80.6\%) belonged to $21-30$ years age group and $72.1 \%$ belonged to Lower socioeconomic status. The mean age of the study population was 25.8 years (SD: 4.1). Overall, 84.7\% consumed mixed diet and 15.3\% had predominant vegetarian diet. Also, $24.2 \%$ subjects were anaemic and among them, only $58.7 \%$ consumed all the IFA tablets given to them. Also $66.8 \%$ of the subjects received the therapy free of cost, since they attended the ANCs in Public Hospitals. In total, $73.2 \%$ of the study population was aware about the importance of IFA tablets.

The overall compliance towards IFA tablets among the subjects was $64.7 \%$. As depicted in Table 1, the compliance was higher among the subjects from lower socioeconomic class, with increasing age and birth, which were found to be statistically significant $(p<0.05)$. The compliance was higher among the subjects with mixed diet. But the difference was not statistically significant. The subjects who received the IFA tablets free of cost had significantly higher compliance $(p=0.029)$ as compared to those from the private hospital who had to spend money for it. 
Table 1: Comparison of socio-demographic factors with the compliance towards IFA tablets among the subjects $(n=190)$

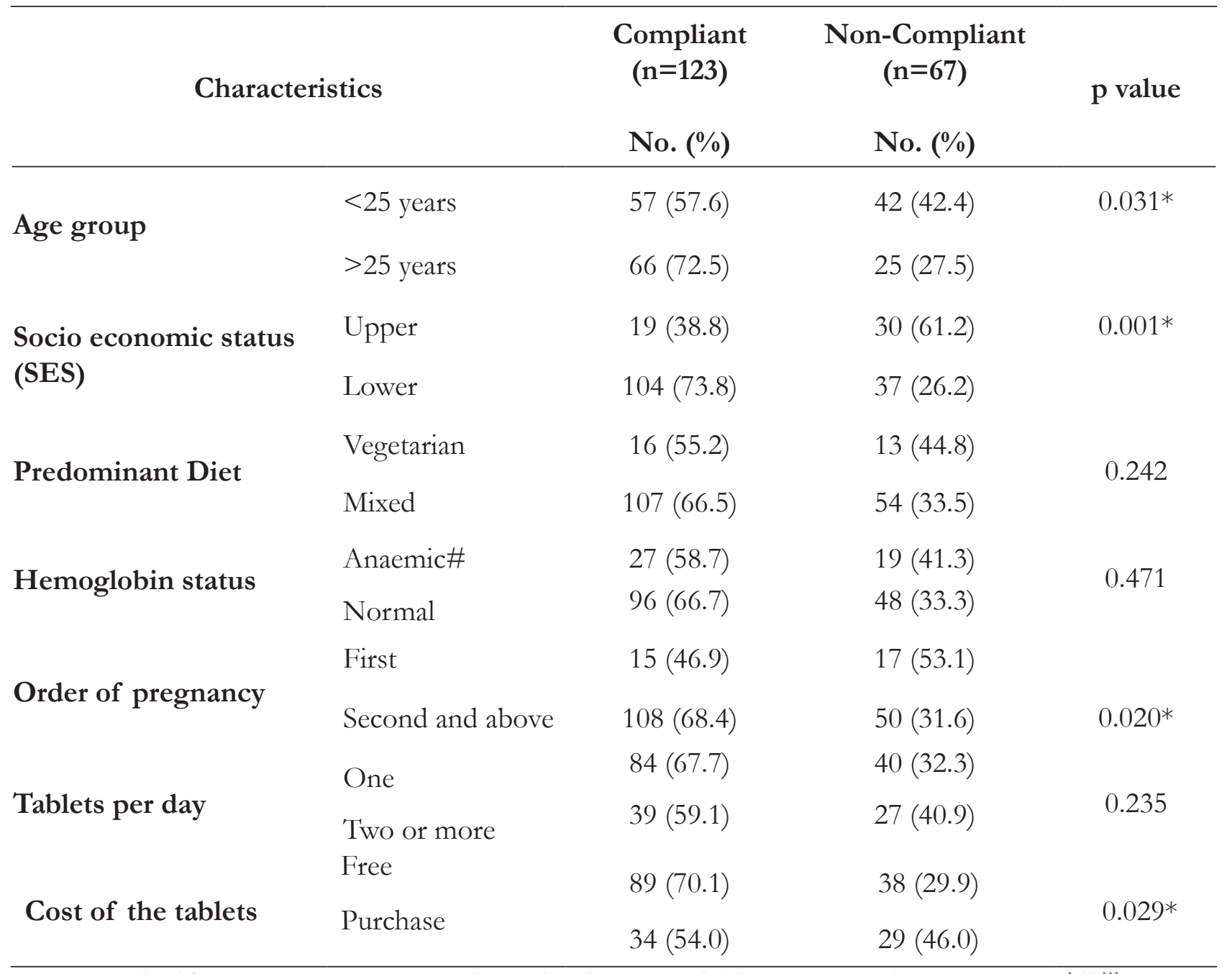

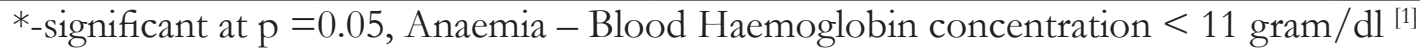

Further, the independent factors contributing towards the compliance were included in the Multiple Logistic Regression analysis and depicted in Table 2. The variables included in the regression analysis were age group, socioeconomic status (SES), predominant diet,
Haemoglobin status, order of pregnancy, number of IFA tablets per day, cost of the IFA tablets. The findings were similar to that of uni-variate analysis. SES, age group, order of pregnancy and cost of IFA tablets had the highest contribution towards the compliance.

Table 3: Reasons for skipping the IFA doses among the subjects $(n=67)$

\begin{tabular}{ll}
\hline Reasons & No. (\%) \\
\hline Forgetfulness & $33(48.8)$ \\
Travel & $09(11.4)$ \\
Constipation & $08(10.1)$ \\
Gastritis & $07(08.9)$ \\
Vomiting & $05(08.6)$ \\
Others & $05(08.6)$ \\
\hline
\end{tabular}


Table 2 Correlates of Compliance to IFA tablets among the subjects: Step-wise multiple logistic regression analysis $(n=190)$

\begin{tabular}{|c|c|c|c|c|c|}
\hline $\begin{array}{l}\text { Correlates of } \\
\text { Compliance }\end{array}$ & $\begin{array}{l}\text { Odds ratio } \\
\text { (Un-adjusted) }\end{array}$ & $95 \% \mathrm{CI}$ & $\begin{array}{l}\text { Odds ratio } \\
\text { (Adjusted) }\end{array}$ & $95 \% \mathrm{CI}$ & $\mathrm{p}$ value \\
\hline \multicolumn{6}{|l|}{ Age group } \\
\hline$<25$ years & 1 & -- & -- & -- & -- \\
\hline$>25$ years & 2.06 & $0.81-3.31$ & 2.39 & $0.94-5.59$ & 0.001 \\
\hline \multicolumn{6}{|c|}{ Socio economic status } \\
\hline Upper & 1 & -- & -- & -- & -- \\
\hline Lower & 6.3 & $2.4-10.2$ & 8.2 & $2.8-13.6$ & $<0.001$ \\
\hline \multicolumn{6}{|l|}{ Predominant Diet } \\
\hline Vegetarian & 1 & -- & -- & -- & -- \\
\hline Mixed & 1.21 & $0.68-2.2$ & 1.9 & $1.10-3.82$ & 0.04 \\
\hline \multicolumn{6}{|c|}{ Hemoglobin status } \\
\hline Anaemic & 1 & -- & -- & -- & -- \\
\hline Normal & 1.32 & $0.79-2.3$ & 1.8 & $1.20-4.12$ & 0.02 \\
\hline \multicolumn{6}{|c|}{ Order of pregnancy } \\
\hline First & 1 & -- & -- & -- & -- \\
\hline Second and above & 4.62 & $0.62-16.34$ & 3.67 & $0.45-20.25$ & $<0.001$ \\
\hline \multicolumn{6}{|l|}{ Tablets per day } \\
\hline Two or more & 1 & -- & -- & -- & -- \\
\hline One & 1.2 & $0.3-2.1$ & 1.5 & $0.4-2.6$ & 0.07 \\
\hline \multicolumn{6}{|l|}{ Cost of the tablets } \\
\hline Purchase & 1 & -- & -- & -- & -- \\
\hline Free & 3.4 & $1.2-5.6$ & 3.7 & $1.6-5.8$ & 0.001 \\
\hline
\end{tabular}

The study subjects also had perceived side-effects with IFA, which included vomiting $(47.69 \%)$, constipation (21.54\%) and gastritis (13.84\%). The reasons for skipping the IFA doses among the non-compliant subjects are given in Table 3. The main reason for skipping the dose is forgetfulness $(48.8 \%)$ followed by travel, constipation, gastritis, vomiting and others.
Among the study subjects, 96.8\% were consuming fruits along with their usual diet. The main reasons for nonconsumption of the fruits among the remainder were the cost of fruits, lack of interest and fear of developing Diabetes Mellitus. Overall, $98.9 \%$ of the subjects were consuming vegetables.

\section{Discussion}

This study found $98 \%$ of the subjects consuming the IFA tablets and compliance was $(64.7 \%)$ which is very 
similar to a study conducted in Senegal in 2008. ${ }^{[8]}$ The reasons for non-compliance as given by the patients in our study include: the experience of side-effects that they associated with the tablets, misunderstanding that they needed to continue taking the tablets throughout pregnancy and forgetfulness which are similar to the previous studies. ${ }^{[2],[4]}$ Additional barriers also could include inadequate counseling and distribution of iron tablets, difficult access and poor utilization of prenatal health care services, beliefs against consuming medications during pregnancy, and in most countries, fears that taking too much iron may cause too much blood or a big baby, making delivery more difficult. ${ }^{[2]}{ }^{[9]}$

According to our study; about $58.1 \%$ subjects consumed all the IFA tablets given to them. This is in accordance with the previous studies done in Karnataka State in 2006 and 2009. ${ }^{[10],[11]}$ The demographic characteristics of the subjects in our study are in accordance with the study conducted by Pena-Rosas et al. ${ }^{[12]}$

Also our study observed that there are direct and indirect factors affecting the compliance levels. Those women, who are anaemic, had lesser level of compliance and the women who were above 25 , and had second pregnancy were more compliant when compared to others. Single doses seemed to have higher IFA compliance levels. This study is very similar to a study conducted among low income group of women in USA during 2005, on pill count adherence to prenatal multivitamin/mineral supplement use in which the researchers reported that ethnicity, null-gravidity, smoking, educational and marital status affected compliance with prescribed medication. [13]. This however needs further evaluation to determine the temporality of association. Add to that the perceived side effects, consciousness about the tablets provided, lack of clarity on the significance of IFA tablets. The present study area reflects a literate population and a prospectively developing region. Despite that there are factors which hinder the compliance of IFA tablets during pregnancy; thereby increasing the chances of skipping the doses. The present study highlights on high proportions of such beliefs and perceptions, similar to the Danish 2012 study. ${ }^{[14]}$

Thus there are many barriers to the successful implementation of IFA therapy among pregnant women. There are fears associated with the side effects of IFA tablets either due to perceived or experienced effects, inadequate counseling and information on significance of IFA tablets in pregnancy and the compliance with them. Reasons like forgetfulness and not being able to take the tablets due to travel are the important patient factors influencing the compliance, which need motivation from the pregnant women and demand further in depth behavioural studies. These socio demographic factors influencing the compliance with IFA tablets and thus determining the anaemia status could still be more and adverse in the other parts of the country where, literacy rates and health care delivery system are yet to reach the desired target. ${ }^{[9]}$

\section{Conclusion}

The study population had a moderate level of compliance towards IFA tablets in pregnancy. The compliance level is directly influenced by the age, socioeconomic status, cost of the IFA tablets and birth order. Also, perceived side effects, lack of clarity on importance of IFA during pregnancy decreased the overall compliance.

\section{Limitations}

The cross sectional nature of the study does not extend to the information on complete iron intake during pregnancy. There were also chances of recall bias among the study subjects regarding skipping of IFA doses.

\section{Recommendations}

During Antenatal visits, the significance of IFA tablets should be repeatedly explained and enforced on. Health education sessions should be conducted for pregnant mothers on regular basis and the same implementation has to be percolated to all cadres of health service providers.

\section{References}

1. Iron deficiency anaemia assessment, prevention and control. Available from URL http://www.who.int/vmnis/indicators/haemoglobin.pdf (Accessed on 10-092012).

2. Galloway R, Dusch E, Elder L, Achadi E, Grajeda R, Hurtado E, et al. Women's perceptions of iron deficiency and anaemia prevention and control in eight developing countries. Social science \& medicine (1982) [Internet]. 2002 Aug ;55(4):529-44. Available from URL: http:/ / www.ncbi.nlm.nih.gov/pubmed/12188461 (Accessed on 1-2-2013).

3. International Institute for Population Sciences (IIPS) and Macro International. 2008. National Family Health Survey (NFHS-3), India, 2005-06: Karnataka. Mumbai: IIPS; 2008. p. 89.

4. Galloway R, McGuire J. Determinants of compliance with iron supplementation: Supplies, side effects, or 
psychology? Social Science \& Medicine 1994; 39:38190.

5. Allen LH. Anaemia and iron deficiency: effects on pregnancy outcome. Am J Clin Nutr 2000;71(suppl):1288S94S.

6. Scholl OT, Johnson WG. Folic acid: influence on the outcome of pregnancy. Am J Clin Nutr 2000; 71(suppl):1295S-303S.

7. Kumar N, Shekhar C, Kumar P, Kundu AS. Kuppuswamy's Socioeconomic Status Scale-Updating for 2007. Indian J Pediatr 2007;74 (12):1131-2.

8. Seck BC, Jackson RT. Determinants of compliance with iron supplementation among pregnant women in Senegal. Public health nutrition [Internet]. 2008 Jun;11(6):596-605. Available from: http://www.ncbi. nlm.nih.gov/pubmed/17764606 (Accessed on 1-22013).

9. Demographic details of Mangalore and other parts of India. Available from URL http:// http://censusindia.gov.in/2011-Common/ahsurvey.html (Accessed on 10-09-2012).

10. Malagi U, Reddy M, Naik RK. Evaluation of National Nutritional Anaemia Control Programme in
Dharwad (Karnataka). J Hum Ecol 2006. 20 (4): 27981.

11. Metgud CS, Katti SM, Mallapur MD, Wantamutte AS. Utilization Patterns of Antenatal Services Among Pregnant Women: A Longitudinal Study in Rural Area of North Karnataka. Al Ameen J Med Sci 2009. 2 (1): 58-62.

12. Peña-Rosas JP, Viteri FE. Effects and safety of preventive oral iron or iron+folic acid supplementation for women during pregnancy. The Cochrane Collaboration and the Cochrane Library 2009. Available from URL http:/ / http:/ / onlinelibrary.wiley.com/ doi/10.1002/14651858.CD004736.pub3/pdf (Accessed on 10-09-2012)

13. Bilimale, A., Anjum, J., Sangolli, H. N., Mallapur, M. Improving adherence to oral iron supplementation during pregnancy. AMJ, 2010, 3, 5, 281-290. Doi 10.4066/ AMJ.2010.291. (Accessed on 7-2-2013).

14. Milman N. Oral Iron Prophylaxis in Pregnancy: Not Too Little and Not Too Much!. Available from URL http:// http://www.hindawi.com/journals/ jp/2012/514345/ (Accessed on 10-09-2012). 